\title{
Previous viral symptoms and individual mothers influenced the leveled duration of human milk antibodies cross-reactive to $S 1$ and S2 subunits from SARS-CoV-2, HCoV-229E, and HCoV-OC43
}

\author{
Veronique Demers-Mathieu (i] ${ }^{1} \cdot$ Ciera DaPra $^{1} \cdot$ Gabrielle B. Mathijssen $^{1} \cdot$ Elena Medo $^{1}$
}

Received: 4 November 2020 / Revised: 29 January 2021 / Accepted: 5 February 2021 / Published online: 1 March 2021

(c) The Author(s), under exclusive licence to Springer Nature America, Inc. 2021

\begin{abstract} $\mathrm{CoV}-2$, and common human coronaviruses $(\mathrm{HCoVs})$ was investigated. viral symptoms. and HCoVs in human milk.

\section{Introduction}

Clinical manifestations of COVID-19 in breastfeeding mothers and their infants are often asymptomatic or mild symptoms [1-4]. The possible transmission of SARS-CoV2 from human milk to the infant is likely rare as 59 human milk samples collected from COVID-19-positive mothers tested negative for SARS-CoV-2 RNA [5]. SARS-CoV-2 was inactive and not infective (not cultivable) when RNA SARS-COV-2 was detected in human milk [6]. The inactivation of SARS-CoV-2 in human milk could be related to the presence of neutralizing antibodies specific to spike (S) protein from SARS-CoV-2.
\end{abstract}

Objective The influence of previous viral symptoms on the level and duration of human milk antibodies reactive to SARS-

Study design Antibodies reactive to S1 and S2 subunits from SARS-CoV-2, HCoV-OC43, and HCoV-229E were measured via ELISA in human milk samples collected from March to June 2020 in mothers with and without viral symptoms.

Results The presence of viral symptoms influenced the levels of SARS-CoV-2 S2-reactive SIgA/IgA and tended to influence SARS-CoV-2 S1 SIgA/IgA and S2-reactive SIgM/IgM in human milk but did not relate to IgG. HCoV-229E S1 + S2reactive $\mathrm{SIgA} / \mathrm{IgA}$ and $\mathrm{SIgM} / \mathrm{IgM}$, as well as $\mathrm{HCoV}-\mathrm{OC} 43 \mathrm{~S} 1+\mathrm{S} 2$-reactive IgG were related to the symptoms. The duration of antibody levels in human milk in mothers with viral symptoms varied between 3 and 4 months post maternal report of

Conclusion Previous viral symptoms and individual mothers may change the antibody cross-reactive levels to SARS-CoV-2

Supplementary information The online version contains supplementary material available at https://doi.org/10.1038/s41372021-01001-0.

Veronique Demers-Mathieu

vdemers-m@medolac.com

1 Department of Neonatal Immunology and Microbiology, Medolac Laboratories A Public Benefit Corporation, Boulder City, NV, USA
Neutralizing antibodies against $\mathrm{S}$ protein can block the viral attachment by binding to the $\mathrm{S} 1$ subunit (virus-receptor binding), fusion by binding to the S2 subunit (virus-cell membrane fusion), and thus, prevent its entry and transmission of human coronaviruses [7]. A recent study demonstrated that mothers with polymerase chain reaction (PCR)-confirmed SARS-CoV-2 had higher levels of antibodies reactive to receptor-binding domain (RBD) SARS-CoV-2 than in nonexposed mothers [8]. These results were predicted as viral infection will usually increase the antibody response in the mammary gland. What is still unknown is how the previous viral symptoms are influencing the antibody responses in mothers. Our recent study demonstrated that the level of $\operatorname{IgG}$ reactive to SARS-CoV-2 S1 + S2 was higher in milk from mothers that had viral symptoms than in milk from mothers without symptoms [9]. Long et al. [10] observed that sera SARS-CoV-2-specific IgG levels in the asymptomatic group were lower relative to the symptomatic group in the acute phase but did not differ for sera IgM. These results suggest that asymptomatic individuals may have a weaker immune response to SARS-CoV-2 infection. The influence of viral symptoms on the antibody levels reactive to $\mathrm{S} 1$ or $\mathrm{S} 2$ from SARS-CoV-2 and their duration across postpartum time in human milk are still unexplored. These questions are essential 
as the strength and duration of immunity after infection are critical factors for "shield immunity".

Most studies on SARS-CoV-2-specific antibodies are from symptomatic patients with severe illness and hospitalization [11-13]. Determining the influence of viral symptoms from mild infection on the production of antibodies reactive to SARS-CoV-2 could underline the importance of preexisting immunity in breastfeeding mothers. Worldwide, fever is the most frequent symptom $(69.4 \%)$, followed by cough $(35 \%)$ in mothers diagnosed COVID-19 by reverse transcriptionpolymerase chain reaction (RT-PCR), whereas $13.4 \%$ of mothers with SARS-CoV-2 infection were asymptomatic [3]. Headaches $(54.9 \%)$, cough $(51.3 \%)$, fever $(39.3 \%)$, sore throat $(34.6 \%)$, and chills $(29.2 \%)$ were the most reported symptoms in mothers with laboratory-confirmed SARS-CoV2 infection in the United States [4].

Another critical question is does preexisting immunity to common human coronavirus $(\mathrm{HCoV})$ strains (causing mild respiratory infections) improves the antibody response against SARS-CoV-2? Children previously infected with $\mathrm{HCoV}-$ OC43 had immunity against HCoV-HKU1 and -229E infection [14]. SARS-COV-2 S-reactive $\mathrm{CD}^{+}{ }^{+}$cells were detected in $35 \%$ of healthy donors that were not diagnosed with COVID-19 [15]. These CD4 ${ }^{+} \mathrm{T}$ cells in healthy donors were mainly active against $\mathrm{S} 2$ and showed a higher homology to $S$ of human coronaviruses compared with $S 1$ [15]. The Tcell lines generated from SARS-Co-2-naive healthy donors responded similarly to $\mathrm{S} 2 \mathrm{HCoV}-229 \mathrm{E}$ and $\mathrm{HCoV}-\mathrm{OC} 43$ [15]. S-protein cross-reactive $\mathrm{T}$ cells were present and may explain the variation of vulnerability to SARS-CoV-2 infection between individuals. Another study reported a range of preexisting memory $\mathrm{CD} 4^{+} \mathrm{T}$ cells that were cross-reactive to SARS-CoV-2, HCoV-OC43, -229E, -NL63, and -HKU1 [16]. Whether the levels of human milk antibodies reactive to SARS-CoV-2 correlate with those reactive to $\mathrm{HCoV}-\mathrm{OC} 43$ and $\mathrm{HCoV}-229 \mathrm{E}$ remain unexplored. A positive correlation could suggest the presence of cross-reactive antibodies and preexisting immunity against SARS-CoV-2.

This study aimed to evaluate the influence of previous viral symptoms on the level and duration of antibodies reactive to SARS-CoV-2, $\mathrm{HCoV}-\mathrm{OC} 43$, and $\mathrm{HCoV}-229 \mathrm{E}$ S1 and S2 subunits in human milk. These results could partially explain the possible preexisting immunity to SARS-CoV-2 due to the cross-reactive antibodies from previous human coronavirus infections.

\section{Materials and methods}

\section{Study design and participants}

Human milk samples were collected every month (once per month for four consecutive months, from March 2020 to June
2020) in five women that had previous viral symptom (between the end of February 2020 and mid-March 2020) and four women that had no symptom of viral infection since the last year. Each donor completed a survey to identify whether they had at least one viral infection related to coronavirus during 2020 (see Table S1 for specific question on the donor survey), when they were sick (including duration and frequency), and what the symptoms were. The screening survey did include an opportunity to share general viral symptoms, many of them related to COVID-19. All donors lived in the United States and were approved donors through Mothers Milk Cooperative (Boulder City, NV). Written consents to use their milk for research were obtained from all participants. Milk collection was approved by the institutional review board (IRB00012424) of Medolac Laboratories. The criteria preestablished for inclusion were passing blood tests (negative tests for HIV, HTLV, hepatitis B or C, and syphilis), living in the United States, and completing a health questionnaire. Mothers who were smokers or drug users were excluded.

\section{Human milk collection}

Human milk samples (150-250 mL) were collected at home with clean electric breast pumps into sterile plastic containers and stored immediately at $-20{ }^{\circ} \mathrm{C}$ in deep freezers. Human milk samples were frozen and transported in insulated boxes to Medolac Laboratories, where they were kept frozen and stored at $-80^{\circ} \mathrm{C}$ until the ELISA measurements.

\section{Human milk antibody levels}

The levels of SIgM/IgM, IgG, and $\mathrm{SIgA} / \operatorname{IgA}$ reactive to SARS-CoV-2 and HCoVs were determined using ELISAs as described in our recent study with some modifications [9]. Briefly, ELISAs were recorded with a microplate reader (SpectraMax iD5, Molecular Devices, Sunnyvale, CA) as reported in our recent studies on human milk antibodies $[17,18]$. Frozen HM samples were rapidly thawed at $37^{\circ}$ and centrifuged at $1301 \times g$ for $20 \mathrm{~min}$ at $4{ }^{\circ} \mathrm{C}$. The supernatant was collected, separated into aliquots, and stored at $-80^{\circ} \mathrm{C}$ until analyzed. Clear flat-bottom microplates were coated with $100 \mu \mathrm{L}$ of recombinant SARS-CoV-2 S1 or S2, HCoV-OC43 $\mathrm{S} 1+\mathrm{S} 2$, or HCoV-229E S1 + S2 (Sino Biological US Inc., Wayne, PA) at $1 \mu \mathrm{g} / \mathrm{mL}$ in $1 \times$ PBS. Microplates were incubated overnight at $4{ }^{\circ} \mathrm{C}$. After incubation, plates were washed three times using PBS $1 \times$ with $0.05 \%$ Tween-20 detergent (PBST), and $200 \mu \mathrm{L}$ of blocking buffer (PBST with $3 \%$ of bovine serum albumin fraction $\mathrm{V}$ ) was added in all wells for $1 \mathrm{~h}$ at room temperature. Standard samples were prepared using milk supernatant with the highest optical density (OD) value for each ELISA (see Figs. S1 and S2). Different standards were used due to the difference of OD values between the different isotypes and the antigens. These standards were 
selected based on the preliminary data of 35 supernatants of human milk diluted at $10 \times$ for $\operatorname{IgG}$ and SIgM/IgM, and $25 \times$ for $\mathrm{SIgA} / \operatorname{IgA}$. The levels of antibodies were derived by interpolation from the standard curves (see Figs. S3 and S4) and assigned quantity expressed in arbitrary units $/ \mathrm{mL}(\mathrm{U} / \mathrm{mL})$. For each step (addition of 100- $\mu \mathrm{L}$ standards/samples and secondary antibodies at $1 \mu \mathrm{g} / \mathrm{mL}$ ), washing and incubation for $1 \mathrm{~h}$ at room temperature were performed. The detection was completed using goat anti-human IgM mu-chain HRP for SIgM/IgM, goat anti-human gamma-chain HRP for IgG, and goat anti-human alpha-chain HRP for SIgA/IgA (Abcam, Cambridge, MA, cat\# ab97205, ab97225, and ab97215).

\section{Statistical analysis}

Two-way ANOVA followed by Tukey's multiplecomparison test was applied using GraphPad Prism (version 8) to compare how the variables' viral symptoms and mothers are related to measured antibody levels. The viral symptoms were the main variable, and the individual mothers were the secondary variable. Multiple comparisons with repeated measures (one-way ANOVA) followed by Tukey (correction) were used to compare how the time during COVID-19 is related to measured antibody levels. The mothers' sample size was determined based on antibody levels detected in our previous studies [9, 19, 20], and proved to be adequately powered based on the results.

\section{Results}

\section{Maternal demographics}

Maternal demographic details of participant groups are presented in Table S2. Postpartum time, infant sex, and maternal age were comparable between the viral symptom group and the no-symptom groups. Individual participant characteristics are presented in Table 1, including the description of the viral infection (symptoms, duration, and date), and postpartum time during milk collection. These maternal factors may influence antibody levels.

\section{Effect of symptoms and mothers on human milk antibody to SARS-CoV-2}

The variation between individual mothers is shown in Fig. 1A-F. The level of SARS-CoV-2 S1-reactive SIgA/ $\operatorname{IgA}$ tended to be influenced by symptoms $(p=0.052)$ (Fig. 1A). S1-reactive SIgM/IgM and IgG were not related to symptoms (Fig. 1C and E).

The level of SARS-CoV-2 S2-reactive SIgA/IgA was influenced by symptoms $(p=0.014$, Fig. 1B). S2reactive $\mathrm{SIgM} / \operatorname{IgM}$ tended to be influenced by symptoms

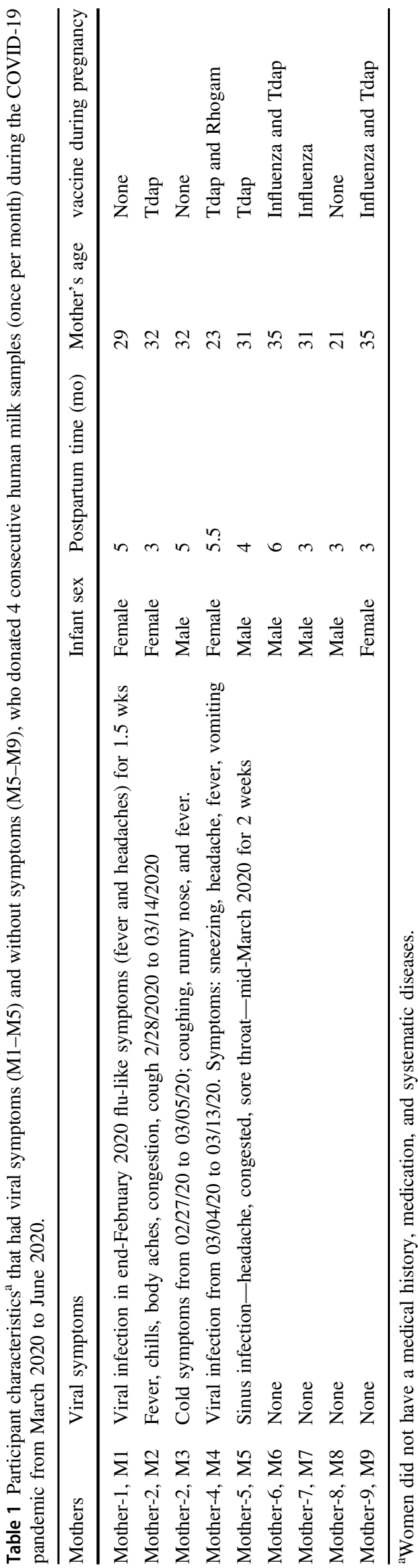




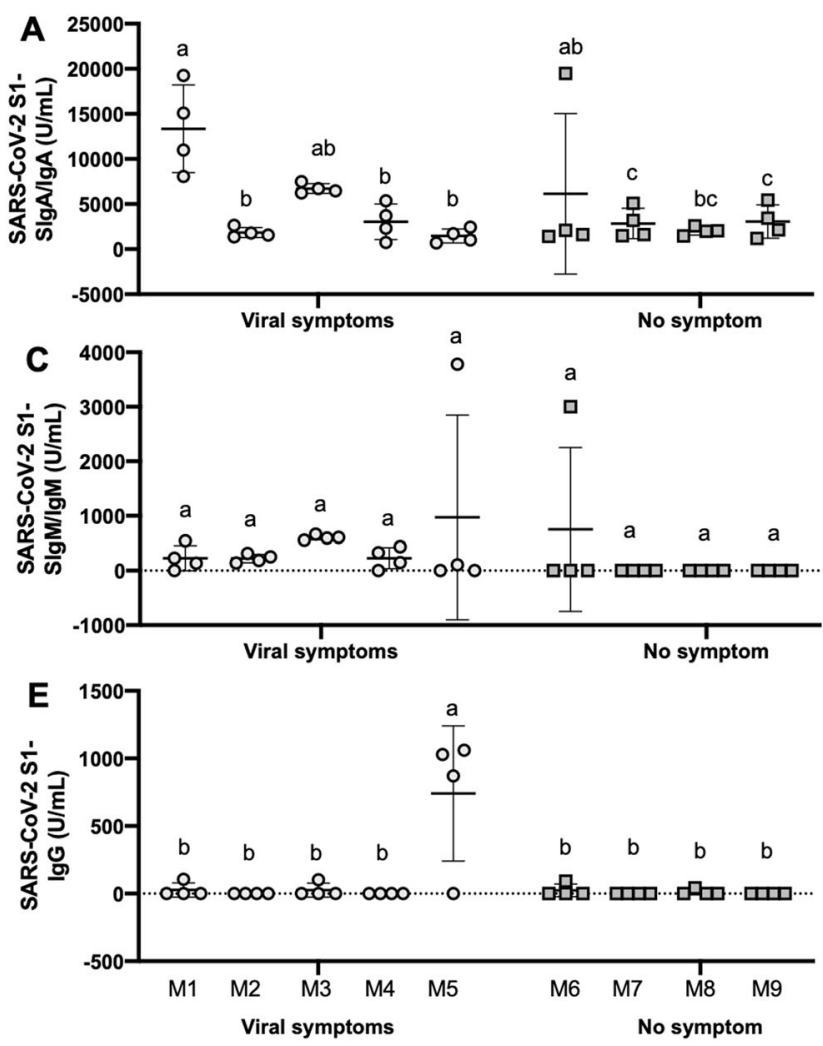

Fig. 1 Influence of viral symptoms on the levels of antibodies reactive to $S 1$ and $S 2$ subunits $S A R S-C o V-2$ in human milk. A S1reactive SIgA/IgA, B S2-reactive SIgA/IgA, C S1-reactive SIgM/IgM, D S2-reactive SIgM/IgM, E S1-reactive IgG, and F S2-reactive IgG. Letters $\mathrm{a}, \mathrm{b}$, and $\mathrm{c}$ show statistically significant differences between

$(p=0.066$, Fig. 1D). S2-reactive $\operatorname{IgG}$ was not related to symptoms (Fig. 1F).

\section{Human milk antibodies across time during COVID-19}

The time (months) during COVID-19 did not influence the overall antibody levels reactive to SARS-CoV-2 in both groups. However, S1-reactive SIgA/IgA was elevated and slowly decreased in milk from mother-1 from March to June (Fig. 2A), whereas S1-reactive SIgM/IgM and IgG were in low levels across postpartum time (Fig. 2B-C). S1and S2-reactive IgG in mother-5 were high level and stable from March to May and declined in June (Figs. 2C and 3C). S1- and S2-reactive SIgM/IgM in mother-5 were stable and low levels during the first three months and strongly increased in June (Figs. 2B and 3B). Most antibodies reactive to $\mathrm{S} 1$ and $\mathrm{S} 2$ in mother-6 (without symptoms) increased from May to June (Figs. 2D-F and 3D-F).

\section{Human milk antibodies reactive to HCoVs}

The variation between individual mothers is shown in Fig. 4A-F. The levels of HCoV-229E S1 + S2-reactive

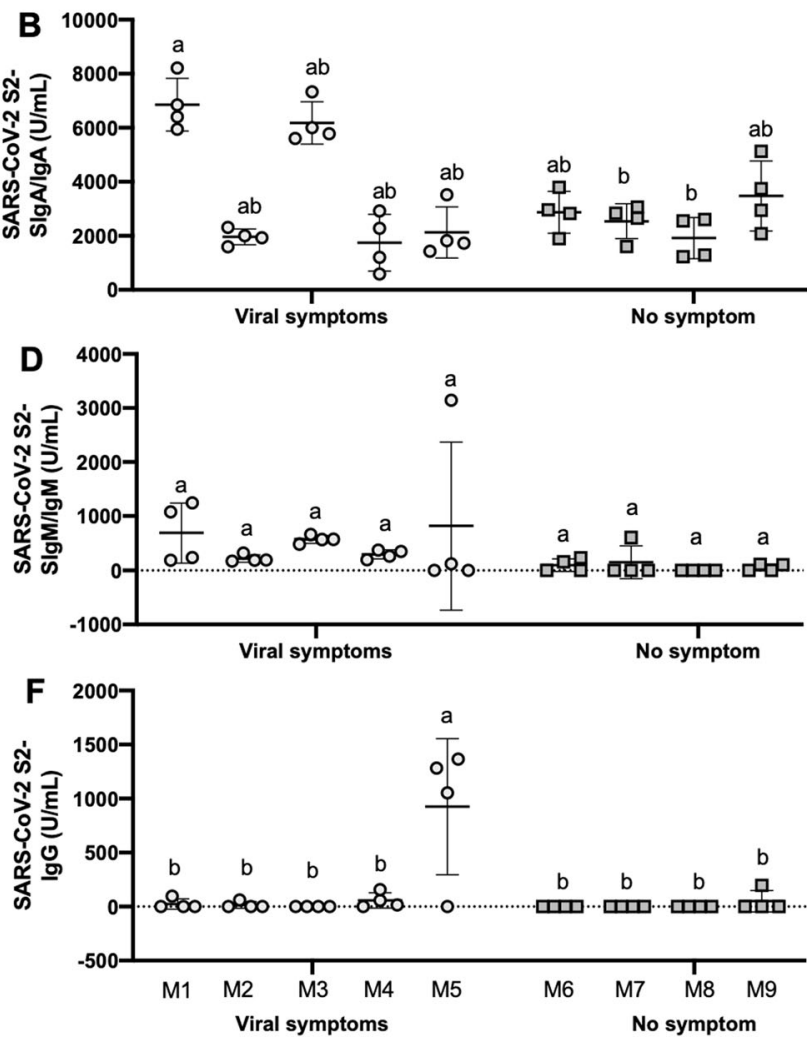

groups $(p<0.05)$ using two-way ANOVA followed by Tukey's multiple-comparison test. Values are means $\pm \mathrm{SD}$, where $n=5$ for mothers in the viral symptom group and $n=4$ for mothers in the nosymptom group. Human milk samples were collected at four different time points across postpartum time for each mother.

SIgA/IgA (Fig. 4A, $p<0.001$ ) and SIgM/IgM (Fig. 4C, $p=$ 0.018 ) were related to the symptoms. HCoV-229E S1 + S2reactive $\operatorname{IgG}$ was influenced by symptoms $(p=0.004)$.

The levels of HCoV-OC43 S1 + S2-reactive SIgA/IgA and SIgM/IgM were not related to symptoms (Fig. 4B, D). HCoV-OC43 S1 + S2-reactive IgG was influenced by symptoms $(p=0.008$, Fig. $4 \mathrm{~F})$.

The time (months) during COVID-19 did not influence the overall levels of $\mathrm{HCoV}$-reactive antibodies in both groups (Figs. S5 and S6). HCoV-229E S1 + S2-reactive SIgM/IgM in mother-1 (symptoms) increased from March to April and decreased progressively in May and June (Fig. S5B). HCoV-229E S1 + S2-reactive IgG in mother-5 (symptoms) was at high levels and stable from March to May and then decreased in June (Fig. S5C).

\section{Discussion}

COVID-19 pandemic formally started in February 2020 in the United States, where confirmed cases rapidly increased to 105,217 cases on $27 / 03 / 20$ [21]. Although the number of laboratory-confirmed cases of SARS-CoV-2 was growing 

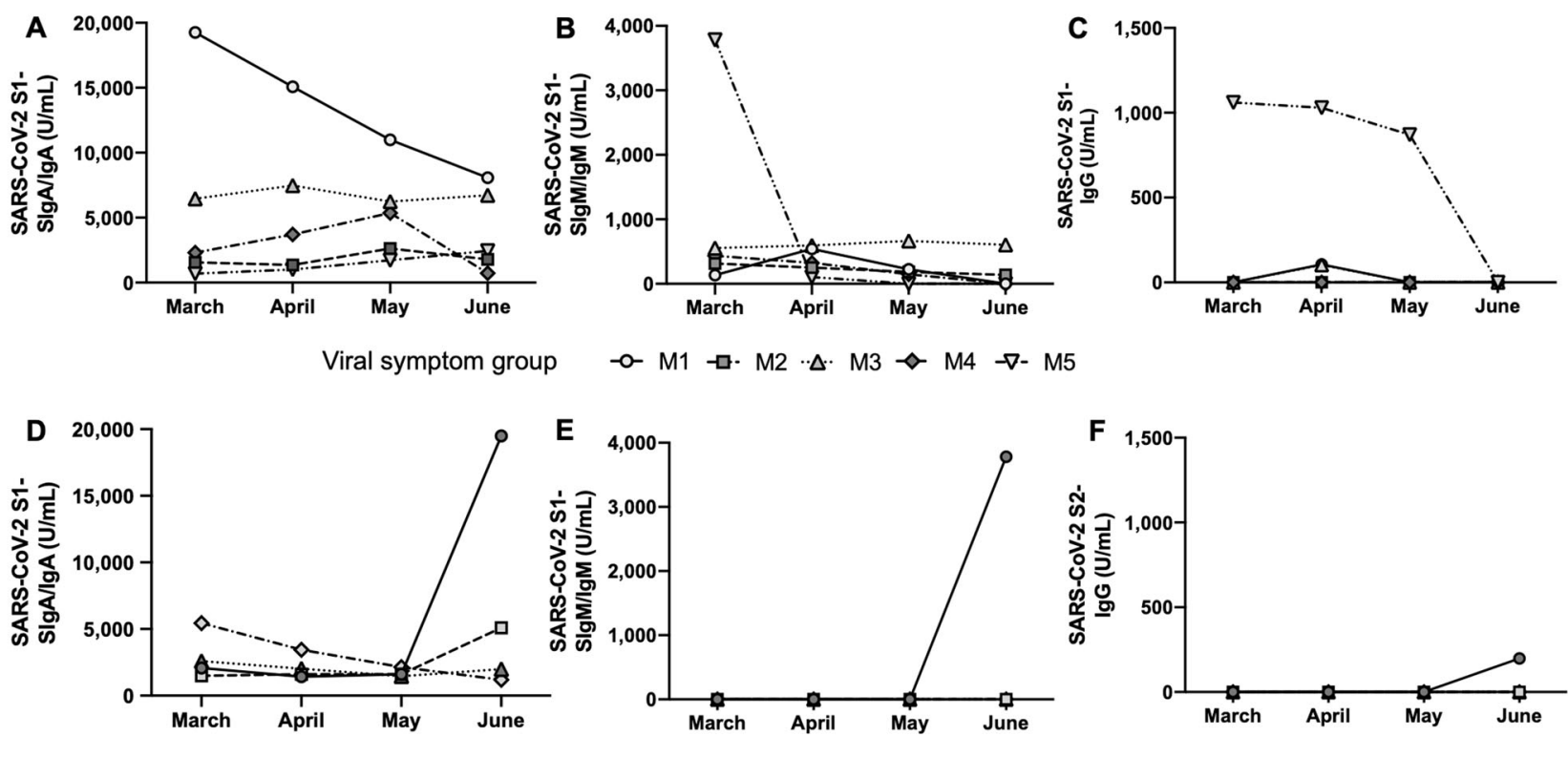

No symptom group

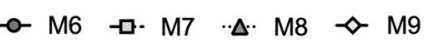

Fig. 2 Changes in the levels of antibodies reactive to S1 SARSCoV-2 in human milk from five mothers with previous viral symptoms and four mothers without symptoms across time during the COVID-19 pandemic. A-C S1-reactive SIgA/IgA, B-D S1reactive SIgM/IgM, C-F S1-reactive $\operatorname{IgG}, \mathbf{A}-\mathbf{C}$ mothers in viral symptom groups, and D-F mothers in the no-symptom group. Human milk samples were collected once per month from March 2020 to June 2020 for each mother (four different time points). M1-M5 are mothers in the viral symptom group, while M6-M9 are mothers in the nosymptom group.
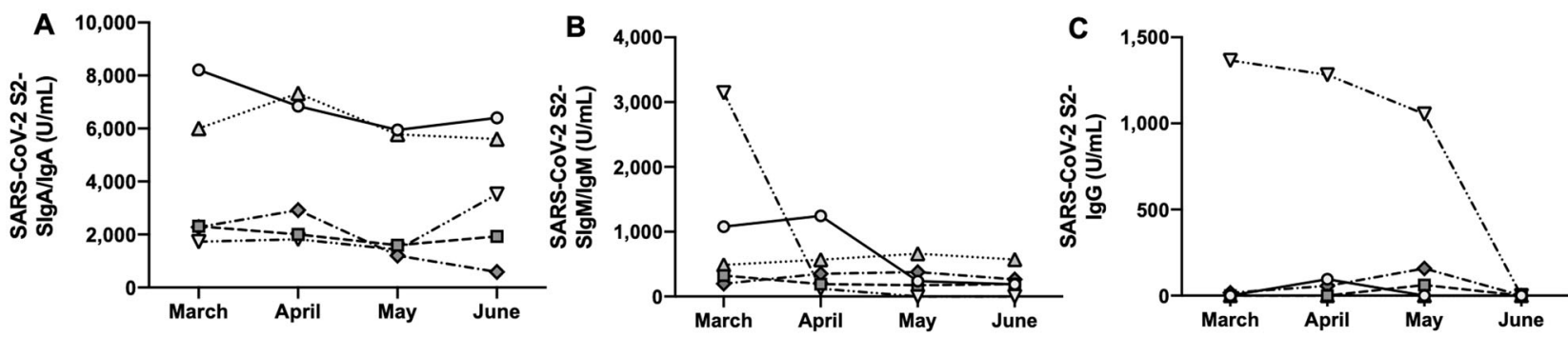

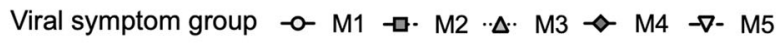
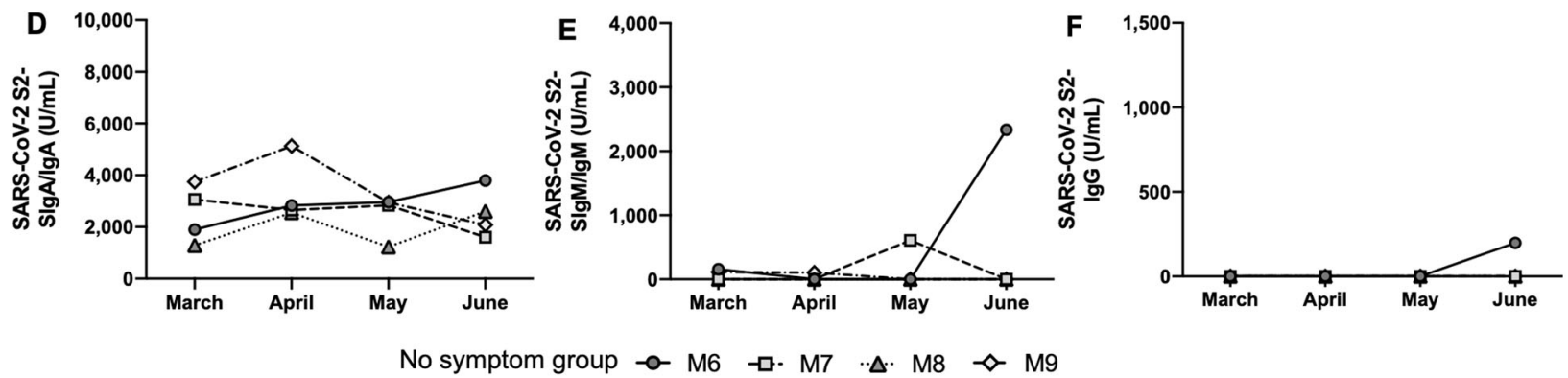

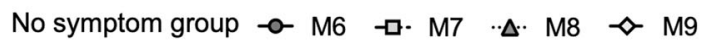

Fig. 3 Changes in the levels of antibodies reactive to S2 SARSCoV-2 in human milk from five mothers with previous viral symptoms and four mothers without symptoms across time during the COVID-19 pandemic. A-C S1-reactive SIgA/IgA, B-D S1reactive $\operatorname{SIgM} / \operatorname{IgM}, \mathbf{C}-\mathbf{F}$ S1-reactive $\mathrm{IgG}, \mathbf{A}-\mathbf{C}$ mothers in viral symptom groups, and D-F mothers in the no-symptom group. Human milk samples were collected once per month from March 2020 to June 2020 for each mother (four different time points). M1-M5 are mothers in the viral symptom group, while M6-M9 are mothers in the nosymptom group.

SARS-CoV-2 infections because testing has been primarily restricted to individuals with moderate-to-severe symptoms due to limited test availability [22]. The quantification of 


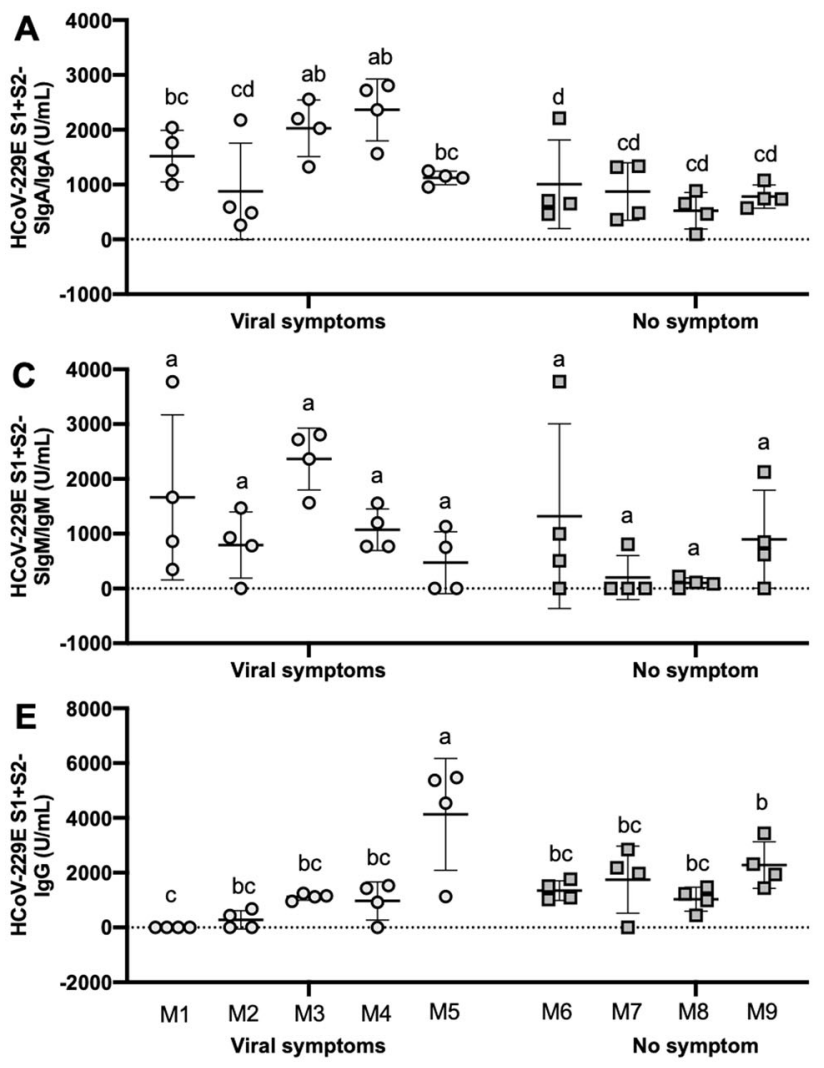

Fig. 4 Influence of viral symptoms on the levels of antibodies reactive to common human coronaviruses (HCoVs) in human milk. A HCoV-229E S1 + S2-reactive SIgA/IgA, B HCoV-OC43 S1 + S2-reactive SIgA/IgA, C HCoV-229E S1 + S2-reactive SIgM/IgM, D HCoV-OC43 S1 + S2-reactive SIgM/IgM, E HCoV-229E S1 + S2reactive $\mathrm{IgG}$, and $\mathbf{F} \mathrm{HCoV}-\mathrm{OC} 43 \mathrm{~S} 1+\mathrm{S} 2$-reactive $\mathrm{IgG}$. Letters a-c

antibodies in undiagnosed mothers is critical to understand the relationship between preexisting immunity, viral symptoms, and antibody responses. This study aimed to evaluate the influence of previous viral symptoms on the level and duration of antibodies reactive to SARS-CoV-2, $\mathrm{HCoV}-229 \mathrm{E}$, and $\mathrm{HCoV}-\mathrm{OC} 43$. The contribution to the field of this study was to analyze coronavirus cross-reactive antibodies in human milk to determine how antibody titers of different viral strains change across four months. The identification of unique differences among antibody titers might be relevant to understand antibody kinetics, preexisting immunity, and passive immunity among maternal and child populations. This area of research is particularly important to determine as infants acquire protection against common coronaviruses and SARS-CoV-2 through human milk.

For the first time, we demonstrated that viral symptoms influenced the levels of SARS-CoV-2 S2-reactive SIgA/IgA and tended to influence SARS-CoV-2 S1-reactive SIgA/IgA and S2-reactive SIgM/IgM in human milk. The presence of symptoms did not relate to SARS-CoV-2- and HCoV-229E-
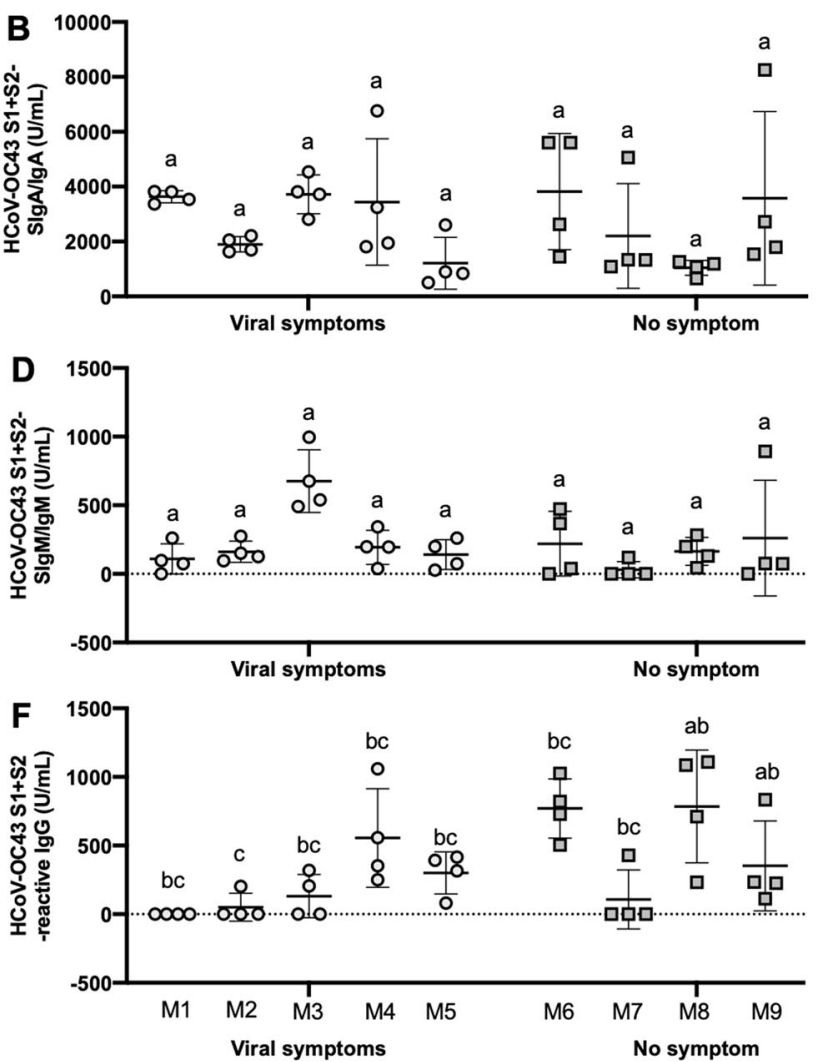

show statistically significant differences between groups $(p<0.05)$ using two-way ANOVA followed by Tukey's multiple-comparison test. Values are means $\pm \mathrm{SD}$, where $n=5$ for mothers in the viral symptom group and $n=4$ for mothers in the no-symptom group. Human milk samples were collected at four different time points across postpartum time for each mother.

reactive $\mathrm{IgG}$ in human milk. On the other hand, $\mathrm{HCoV}$ 229E S1 + S2-reactive SIgA/IgA and SIgM/IgM, as well as HCoV-OC43 S1 + S2-reactive IgG were related to viral symptoms. Our results suggest that human milk antibodies may be cross-reactive to multiple, but not all viruses. These observations are in agreement with recent publications suggesting that immunity to "common cold" coronaviruses [16] or other viruses [23] may produce cross-reactive antibodies to SARS-CoV-2. Mateus et al. [16] demonstrated a range of preexisting memory $\mathrm{CD}_{4}^{+} \mathrm{T}$ cells that were crossreactive to SARS-CoV-2, $\mathrm{HCoV}-\mathrm{OC} 43, \mathrm{HCoV}-229 \mathrm{E}$, HCoV-NL63, and HCoV-HKU1. Shrock et al. [23] showed that patients who required hospitalization during COVID-19 infection exhibited stronger and broader antibody responses to SARS-CoV-2 but weaker overall responses to past infections with common coronaviruses compared with patients who did not need hospitalization. The hospitalized group had higher seroprevalence rates for cytomegalovirus and herpes simplex virus 1 . These studies suggest that other viral exposures could provide cross-reactive protection against SARS-CoV-2 disease instead of specific. 
More than $90 \%$ of the human population is seropositive for at least three HCoVs [24]. SARS-CoV-2-reactive T cells in individuals unexposed to SARS-CoV-2 were likely derived from exposure to $\mathrm{HCoVs}$ (including $\mathrm{HCoV}-229 \mathrm{E}$ and HCoV-OC43) [25, 26]. S-reactive T cells in patients with COVID-19 responded similarly to HCoV-229E S and SARS-CoV-2 S, suggesting that S-cross-reactive T cells' presence was probably generated during past encounters with endemic coronaviruses [15]. Preexisting SARS-CoV2-reactive antibodies could be relevant because individuals with a high level of preexisting antibodies recognizing SARS-CoV-2 could mount a faster and more robust immune response during exposure to SARS-CoV-2, thus limiting COVID-19 disease severity [25].

$\mathrm{SIgA} / \mathrm{IgA}$ reactive to SARS-CoV-2 S1 and S2 in milk from mother-1 was the highest level among most mothers, whereas IgG reactive to SARS-CoV-2 S1 and S2 was the highest in milk from mother-5. Mother- 1 and mother-5 may have been infected by a strain of human coronaviruses or other viruses due to their high antibody levels. These influenza-unvaccinated mothers had viral symptoms around February-March 2020, which could be related to other infections, including influenza outbreaks. Whether these women were exposed to SARS-CoV-2 remains unknown. In this study, the production of isotype reactive to SARSCoV-2 varied greatly between mothers. These observations agree with Long et al. [10], reporting a significant variation of sera IgG and IgM levels specific to SARS-CoV-2 S between patients in symptomatic groups collected during the convalescent phase COVID-19. In this study, the duration of antibody levels in human milk varied between 3 and 4 months after mothers reported viral symptoms. Recent studies $[10,27,28]$ showed that sera IgG is secreted after seven days and slowly decreased after $~ 2-3$ months. Sera S-specific IgA was the highest at 20-22 days post infection and was stable until 42 days [29]. Dong et al. [30] showed that women with confirmed PCR test had IgG and IgA detected in human milk after two weeks of post infection and the highest antibody levels after five weeks. Her infant had negative PCR results for RNA SARS-CoV-2 at birth but had elevated IgG in serum, suggesting that infants can acquire passive immunity against SARS-CoV-2 by ingesting human milk from mothers recovering from COVID-19. Individual mothers likely influence the kinetics of human milk antibodies due to the differences of previous infections (time since disease onset and severity), preexisting immunity, age, genetic factors, and other factors affecting the immune response [18, 31].

Our study has some limitations. First, human milk antibodies were not evaluated using neutralization assays, and their neutralizing capacities remain to be determined. Second, the demographic description of the participants is limited and might be subjected to systematic bias. Maternal factors, including socioeconomic status, race, preexisting immunity conditions, genetic factors, and nutritional status, can affect the antibody levels due to their direct or indirect impact on passive immunity. Third, no direct clinical assessment of coronavirus infection was performed using a confirmed diagnostic of RNA SARS-CoV-2. The US Center for Disease Control and Prevention had to withdraw testing kits in March 2020, when they were shown to have a high rate of false positives due to reagent contamination [32]. The symptoms described by the participants could be from the infection to strains of human coronavirus or any other viruses. Finally, it is unlikely that the lactation time affected antibody levels in human milk reactive to SARSCoV-2 as all samples were collected between 3 and 5.5 months of postpartum time. Our previous study demonstrated that the antibody concentrations were not affected by the postpartum time when human milk collection was collected after 4 months of lactation [18].

\section{Conclusion}

Our study reveals the presence of antibodies cross-reactive to SARS-CoV-2, HCoV-229E, and HCoV-OC43 S1 and $\mathrm{S} 2$ subunits in human milk. The presence of viral symptoms in mothers influenced the levels of SARS-CoV-2 S2reactive $\mathrm{SIgA} / \operatorname{Ig} \mathrm{A}$ and tended to influence the levels of SARS-CoV-2 S1 SIgA/IgA and S2-reactive SIgM/IgM in human milk but did not relate to $\mathrm{IgG}$. The duration of antibody levels in human milk in mothers with viral symptoms varied between 3 and 4 months post maternal report of viral symptoms. This study underlines the importance of breastfeeding to provide passive immunity to infants via human milk antibodies. The next step of this investigation will be to determine the neutralizing capacity of human milk antibodies against SARS-CoV-2 spikepseudotyped lentiviral particles and replication-competent live SARS-CoV-2. Neutralizing antibodies are generally correlated with protection against reinfection [33]. Neutralizing antibodies against SARS-CoV-2 could prevent and treat COVID-19 in infants, but also failed individuals with SARS-CoV-2 vaccine and people at risk, including immunocompromised patients, pregnant women, children, and older people. Finally, future study is needed to evaluate the presence of cross-reactive antibodies between SARS-CoV-2 and other common viruses, such as influenza viruses or rhinoviruses.

Acknowledgements We thank all study participants for their valuable contributions.

Funding The authors (VDM, CD, GBM, and ED) disclosed receipt of the financial support from Medolac Laboratories A Public Benefit Corporation for the conduct of the study. 
Disclosure VDM, DMD, GM, and EM are employees at Medolac Laboratories.

Author contributions VDM conceptualized and designed the study, carried out the statistical analysis, drafted the paper, and approved the final paper as submitted. VDM has primary responsibility for the final content. CD and GBM carried out ELISA analyses. EM acquired funding for this study. CD, GBM, and EM revised the paper and approved the final paper as submitted.

\section{Compliance with ethical standards}

Conflict of interest VDM, CD, GBM, and EM are employees at Medolac Laboratories.

Publisher's note Springer Nature remains neutral with regard to jurisdictional claims in published maps and institutional affiliations.

\section{References}

1. Chang TH, Wu JL, Chang LY. Clinical characteristics and diagnostic challenges of pediatric COVID-19: a systematic review and meta-analysis. J Formos Med Assoc. 2020;119:982-9.

2. Xia W, Shao J, Guo Y, Peng X, Li Z, Hu D. Clinical and CT features in pediatric patients with COVID-19 infection: different points from adults. Pediatr Pulmonol. 2020;55:1169-74.

3. Bellos I, Pandita A, Panza R. Maternal and perinatal outcomes in pregnant women infected by SARS-CoV-2: a meta-analysis. Eur J Obstet Gyn Reprod Biol. 2020;256:194-204.

4. Zambrano LD, Ellington S, Strid P, Galang RR, Oduyebo T, Tong VT, et al. Update: characteristics of symptomatic women of reproductive age with laboratory-confirmed SARS-CoV-2 infection by pregnancy status-United States, January 22-October 3, 2020. Morb Mortal Wkly Rep. 2020;69:1641.

5. Centeno-Tablante E, Medina-Rivera M, Finkelstein JL, RaycoSolon P, Garcia-Casal MN, Rogers L, et al. Transmission of SARS-CoV-2 through breast milk and breastfeeding: a living systematic review. Ann N Y Acad Sci. 2020. https://doi.org/10. 1111/nyas. 14477 .

6. Chambers C, Krogstad P, Bertrand K, Contreras D, Tobin NH, Bode L, et al. Evaluation for SARS-CoV-2 in breast milk from 18 infected women. J Am Med Assoc. 2020;324:1347-8.

7. Jiang S, Hillyer C, Du L. Neutralizing antibodies against SARSCoV-2 and other human coronaviruses. Trends Immunol. 2020; 41:355-9.

8. Fox A, Marino J, Amanat F, Krammer F, Hahn-Holbrook J, ZollaPazner S, et al. Robust and specific secretory IgA against SARSCoV-2 detected in human milk. IScience. 2020;23:101735.

9. Demers-Mathieu V, Do DM, Mathijssen GB, Sela DA, Seppo A, Järvinen KM, et al. Difference in levels of SARS-CoV-2 S1 and S2 subunits- and nucleocapsid protein-reactive SIgM/IgM, IgG and SIgA/IgA antibodies in human milk. J Perinatol. 2020;1 (Sept):1-10.

10. Long QX, Tang XJ, Shi QL, Li Q, Deng HJ, Yuan J, et al. Clinical and immunological assessment of asymptomatic SARS-CoV-2 infections. Nat Med. 2020;26:1200-4.

11. Liu L, Wang P, Nair MS, Yu J, Rapp M, Wang Q, et al. Potent neutralizing antibodies against multiple epitopes on SARS-CoV-2 spike. Nature. 2020;584:450-6.

12. Zost SJ, Gilchuk P, Chen R, Case JB, Reidy JX, Trivette A, et al. Rapid isolation and profiling of a diverse panel of human monoclonal antibodies targeting the SARS-CoV-2 spike protein. Nat Med. 2020;26:1422-7.
13. Gao X, Wang S, Zeng W, Chen S, Wu J, Lin X, et al. Clinical and immunologic features among COVID-19-affected mother-infant pairs: antibodies to SARS-CoV-2 detected in breast milk. N. Microbes N. Infect. 2020;37:100752.

14. Dijkman R, Jebbink MF, Gaunt E, Rossen JW, Templeton KE, Kuijpers TW, et al. The dominance of human coronavirus OC43 and NL63 infections in infants. J Clin Virol. 2012;53:135-9.

15. Braun J, Loyal L, Frentsch M, Wendisch D, Georg P, Kurth F, et al. SARS-CoV-2-reactive T cells in healthy donors and patients with COVID-19. Nature. 2020;29:1-5.

16. Mateus J, Grifoni A, Tarke A, Sidney J, Ramirez SI, Dan JM, et al. Selective and cross-reactive SARS-CoV-2 T cell epitopes in unexposed humans. Science. 2020;370:89-94.

17. Demers-Mathieu V, Mathijssen GB, DaPra C, Medo E. The effects of probiotic supplementation on the gene expressions of immune cell surface markers and levels of antibodies and proinflammatory cytokines in human milk. J Perinatol. 2020;18:1-9.

18. Demers-Mathieu V, Mathijssen GB, DaPra C, Do DM, Medo E. Active free secretory component and secretory $\operatorname{IgA}$ in human milk: do maternal vaccination, allergy, infection, mode of delivery, nutrition and active lifestyle change their concentrations? Pediatr Res. 2020;20:1-8.

19. Demers-Mathieu V, Huston RK, Markell AM, McCulley EA, Martin RL, Dallas DC. Impact of pertussis-specific IgA, IgM, and IgG antibodies in mother's own breast milk and donor breast milk during preterm infant digestion. Pediatr Res. 2020;29:1-8.

20. Demers-Mathieu V, Huston RK, Markell AM, McCulley EA, Martin RL, Dallas DC. Antenatal influenza A-specific IgA, IgM, and IgG antibodies in mother's own breast milk and donor breast milk, and gastric contents and stools from preterm infants. Nutrients. 2019;11:1567.

21. COVID-19 Dashboard by the CSSE at Johns Hopkins University (JHU). 2020. https://coronavirus.jhu.edu/map.html.

22. Wu F, Wang A, Liu M, Wang Q, Chen J, Xia S, et al. Neutralizing antibody responses to SARS-CoV-2 in a COVID-19 recovered patient cohort and their implications. Lancet. 2020. https://doi.org/ 10.2139/ssrn.3566211

23. Shrock E, Fujimura E, Kula T, Timms RT, Lee IH, Leng Y, et al. Viral epitope profiling of COVID-19 patients reveals crossreactivity and correlates of severity. Science 2020;370:1-15.

24. Gorse GJ, Patel GB, Vitale JN, O'Connor TZ. Prevalence of antibodies to four human coronaviruses is lower in nasal secretions than in serum. Clin Vaccin Immunol. 2010;17:1875-80.

25. Sette A, Crotty S. Pre-existing immunity to SARS-CoV-2: the knowns and unknowns. Nat Rev Immunol. 2020;20:457-8.

26. Grifoni A, Weiskopf D, Ramirez SI, Mateus J, Dan JM, Moderbacher CR, et al. Targets of T cell responses to SARS-CoV-2 coronavirus in humans with COVID-19 disease and unexposed individuals. Cell. 2020;181:1489-501.

27. Long QX, Liu BZ, Deng HJ, Wu GC, Deng K, Chen YK, et al. Antibody responses to SARS-CoV-2 in patients with COVID-19. Nat Med. 2020;29:1-4.

28. Wang K, Long QX, Deng HJ, Hu J, Gao QZ, Zhang GJ, et al. Longitudinal dynamics of the neutralizing antibody response to SARS-CoV-2 infection. Clin Infect Dis. 2020. https://doi.org/10. 1093/cid/ciaa1143.

29. Padoan A, Sciacovelli L, Basso D, Negrini D, Zuin S, Cosma C, et al. IgA-Ab response to spike glycoprotein of SARS-CoV-2 in patients with COVID-19: a longitudinal study. Clin Chim Acta. 2020;507:164-6.

30. Dong Y, Chi X, Hai H, Sun L, Zhang M, Xie WF. et al. Antibodies in the breast milk of a maternal woman with COVID-19. Emerg Microbes Infect. 2020;9:1467-9.

31. Huang AT, Garcia-Carreras B, Hitchings MD, Yang B, Katzelnick LC, Rattigan SM, et al. A systematic review of antibody 
mediated immunity to coronaviruses: kinetics, correlates of protection, and association with severity. Nat Commun. 2020;11 (Sep 17):1-6.

32. Willman D Contamination at CDC lab delayed rollout of coronavirus tests. The Washington Post. 2020. https://www.wa shingtonpost.com/investigations/contamination-at-cdc-lab-delayed- rollout-of-coronavirus- tests/2020/04/18/fd7d3824-7139-11ea-aa80c2470c6b2034_story.html.

33. Crawford KH, Eguia R, Dingens AS, Loes AN, Malone KD, Wolf CR, et al. Protocol and reagents for pseudotyping lentiviral particles with SARS-CoV-2 Spike protein for neutralization assays. Viruses. 2020;12:513. 